\title{
POLARIZATION OF IRAS QUASARS AND THE INNER STRUCTURE OF ACTIVE GALACTIC NUCLEI
}

\author{
Beverley J. Wills, ${ }^{1}$ D. Wills, ${ }^{1}$ N.J. Evans, ${ }^{1}$ A. Natta, ${ }^{2}$ K.L. Thompson, ${ }^{1}$ \\ M. Breger, ${ }^{1,3}$ M.L. Sitko, ${ }^{4}$ D.F. Lester, ${ }^{1}$ D.R. Garnett, ${ }^{1}$ and S.R. Sawyer ${ }^{1}$
}

We have found that 4 new, bright IRAS quasars, out of 7 observed, have strong, nonvariable, wavelength-dependent polarization. Three show degrees of polarization, $p_{\lambda}$, increasing from infrared to UV wavelengths (Fig. 1), which implies a combination of a polarized, scattered spectrum and a much redder, unpolarized spectrum. Detailed IR and optical polarimetry and spectrophotometry of one, IRAS 13349+2438 (Wills et al.), shows a polarized flux spectrum, $\mathrm{p}_{\lambda} \times \mathrm{F}_{\lambda}$, (continuum and $\mathrm{Pa} \alpha, \mathrm{H} \alpha$, and $\mathrm{H} \beta$ broad hydrogen lines) typical of unreddened, luminous quasars. This suggests that the path of scattered light from a central, luminous quasar is low in dust and that the polarization of the scattered spectrum is wavelength independent. The latter is most easily explained by electron scattering although the data do not exclude dust scattering. When this polarized flux spectrum is subtracted from the total spectrum, we are left with a very reddened line and continuum spectrum, $E(B-V)=0.3$ to 0.7 , which we attribute to the same luminous quasar seen through a thick dusty torus. The angle of polarization is parallel to the major axis of the r-band image, presumed to be that of the host galaxy. If the torus is in the plane of the galaxy, the axial ratio suggests a viewing angle of $40^{\circ}$ to the plane of the torus. Fig. 2 illustrates the geometry. The appearance of the quasar at optical and UV wavelengths will depend strongly on viewing angle, suggesting that present samples of quasars selected by colours, optical flux density, or quasi-stellar appearance, may be seriously biased, with important consequences for studies of the space density and evolution of AGN.

Our model can be related to the physical model proposed by Krolik and Begelman to explain the polarized broad emission line spectrum of NGC 1068 (Miller, these proceedings). These observations and models place IRAS quasars within the evolutionary scheme proposed by Sanders $e t$ al. If these schemes are correct, inclination to the line-ofsight, and dust content (related to evolutionary age) are key parameters that determine the appearance of an AGN.

\section{References}

Krolik, J.H., and Begelman, M.C. 1986, Ap.J. (Letters), 308, L55; 1988, Ap.J., in press.

Sanders, D.B., et al. 1988, Ap.J., 325, 74; and these proceedings.

Wills, B.J., et al. 1988, Ap.J., submitted.

${ }^{1}$ Department of Astronomy, University of Texas at Austin, TX 78712, USA

2Osservatorio di Arcetri, Largo E.Fermi 5, 50125, Firenze, Italy

3University of Vienna Observatory, Turkenschanzstrasse 17, A 1180 Vienna, Austria

${ }^{4}$ Department of Physics, University of Cincinatti, OH 34221-0011, USA 

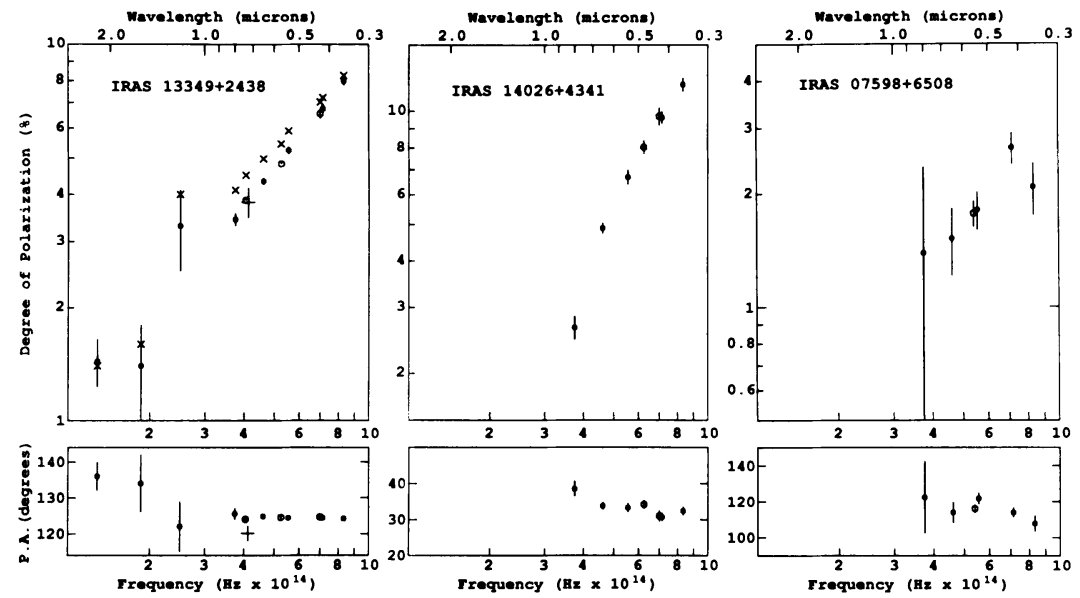

Fig. 1: The inverse wavelength dependence of linear polarization through UBVRI(JHK) bands. The + represents the polarization of the broad $\mathrm{H} \alpha$ emission line, and the $\mathrm{x}$ 's the polarization after correction for the host galaxy, assumed unpolarized.

Pole-on view: A normal, high luminosity QSO.

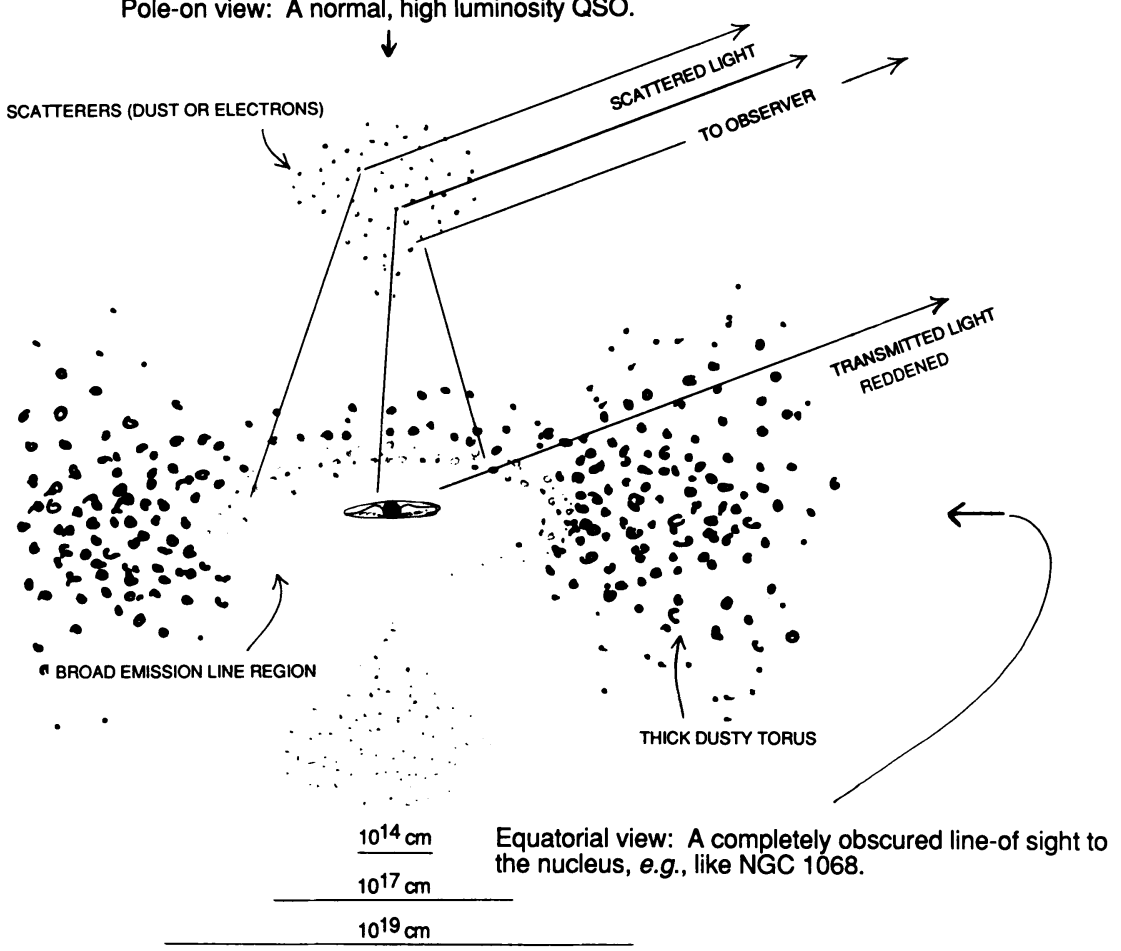

Fig. 2: A cross-section through a possible model for IRAS 13349+2438 (not to scale). 